\title{
ASYMPTOTIC ANALYSIS OF SINGULAR SOLUTIONS OF THE SCALAR AND MEAN CURVATURE EQUATIONS
}

\author{
GONZALO GARCÍA AND HENDEL YAKER
}

Received 21 October 2003 and in revised form 18 January 2005

We show that positive solutions of a semilinear elliptic problem in the Sobolev critical exponent with Newmann conditions, related to conformal deformation of metrics in $\mathbb{R}_{+}^{n}$, are asymptotically symmetric in a neighborhood of the origin. As a consequence, we prove for a related problem of conformal deformation of metrics in $\mathbb{R}_{+}^{n}$ that if a solution satisfies a Kazdan-Warner-type identity, then the conformal metric can be realized as a smooth metric on $S_{+}^{n}$.

\section{Introduction}

In recent years there has been a huge interest in studying properties of the positive singular solutions $u$ of the scalar curvature equation

$$
\begin{gathered}
\Delta u+k(x) u^{(n+2) /(n-2)}=0 \quad \text { in } B_{1} \backslash\{0\} \\
u(x)>0, \quad u \in \mathbf{C}^{2}\left(B_{1} \backslash\{0\}\right),
\end{gathered}
$$

where $k(x)$ is a smooth positive function and $B_{1}$ is the unit ball of $\mathbb{R}^{n}$, with $n \geq 3$.

We observe that the interest for studying singular solutions of (1.1) comes from the study of asymptotic behavior of positive solutions of the following problem in $\mathbb{R}^{n}, n \geq 3$ :

$$
\Delta v+\tilde{k}(x) v^{(n+2) /(n-2)}=0 \quad \text { in } \mathbb{R}^{n}
$$

Using the Kelvin transformation, the study of the asymptotic behavior of solutions $v$ of problem (1.2) at infinity is reduced to the study of the asymptotic behavior at zero of solutions of problem (1.1).

For $k(x) \equiv 1$, Caffarelli et al. in [2] proved that if zero is a nonremovable singularity of a solution $u$ of (1.1), then $u$ is asymptotically symmetric with respect to the origin. Furthermore, they showed the existence of a radial solution $u_{0}$ of the problem

$$
\begin{gathered}
\Delta u_{0}+u_{0}^{(n+2) /(n-2)}(x)=0, \quad u_{0}>0 \quad \text { in } \mathbb{R}^{n} \backslash\{0\}, \\
u_{0} \in \mathbf{C}^{2}\left(\mathbb{R}^{n} \backslash\{0\}\right), \quad \lim _{x \rightarrow 0} u_{0}(x)=+\infty,
\end{gathered}
$$


such that

$$
u(x)=u_{0}(x)(1+o(1))
$$

as $x \rightarrow 0$. A consequence of (1.4) is that for any solution $u$ of (1.3), there exist positive constants $c_{1}, c_{2}$ such that

$$
c_{1}|x|^{(2-n) / 2} \leq u(x) \leq c_{2}|x|^{(2-n) / 2} .
$$

In case $k(x)$ is a nonconstant function, Chen and Lin extended these results in [4] by assuming that

$$
c_{1}|x|^{l-1} \leq|\nabla k(x)| \leq c_{2}|x|^{l-1},
$$

where $l, c_{1}, c_{2}$ are positive constants. They showed in [3] that if the previous inequalities hold for some $l \geq(n-2) / 2$, then $u$ satisfies the upper bound of (1.5),

$$
u(x) \leq c|x|^{(2-n) / 2},
$$

for any solution of (1.1) in a neighborhood of the origin, where $c$ is a positive constant. They established in [4] that the asymptotic symmetry for solutions of (1.1) follows from (1.7).

In this paper, we are interested in studying singular solutions of an elliptic problem related to conformal deformation of metrics in $\mathbb{R}_{+}^{n}$. We will use the methods of Chen and Lin in [4] to study the positive singular solutions $u \in \mathbf{C}^{2}\left(B_{1}^{+}\right) \cap \mathbf{C}^{1}\left(\partial B_{1}^{+} \backslash\{0\}\right)$ of the scalar and mean curvature equations

$$
\begin{gathered}
\Delta u+k(x) u^{(n+2) /(n-2)}=0 \quad \text { in } B_{1}^{+}, \\
\frac{\partial u}{\partial \eta}=0 \quad \text { in } \partial^{\prime} B_{1}^{+} \backslash\{0\},
\end{gathered}
$$

where $n \geq 3, B_{1}^{+}=B_{1} \cap \mathbb{R}_{+}^{n}, \partial^{\prime} B_{1}^{+}=\partial B_{1}^{+} \cap \partial \mathbb{R}_{+}^{n}$, and $\eta$ denotes the normal outward unit vector in the boundary.

We assume that $k \in \mathbf{C}^{1}\left(\bar{B}_{1}^{+}\right)$is bounded between two positive constants and satisfies, in a neighborhood of the origin,

$$
c_{1}|x|^{l-1} \leq|\nabla k(x)| \leq c_{2}|x|^{l-1},
$$

where $l, c_{1}, c_{2}$ are positive constants. 
A motivation in studying problem (1.8) arises from the problem of finding a metric, conformal to the Euclidean $\mathbb{R}_{+}^{n}$, such that $k(x)$ is the scalar curvature and zero is the mean curvature of the new metric. Our first observation is that if $\partial k / \partial \eta=0$ in $\partial^{\prime} B_{1}^{+}$, then $k$ can be extended to a function $\tilde{k} \in \mathbf{C}^{1}\left(\bar{B}_{1}\right)$. Moreover, any solution $u$ of problem (1.8) can be extended to a solution of problem (1.1) in the punctured ball $B_{1} \backslash\{0\}$. If (1.9) holds for some $l \geq(n-2) / 2$, then $u$ satisfies (1.7).

According to the previous discussion, we are going to assume condition (1.7) on the solutions $u$ of problem (1.8). We point out that Chen and Lin in [5] conjectured that if $k$ is Hölder continuous, then any solution of (1.1) satisfies (1.7).

In order to present the results, we start by defining the numbers $P(r, u)$ and $D(u)$ associated with a solution $u$ of problem (1.8), as follows:

$$
P(r, u)=\int_{\partial^{\prime \prime} B_{r}^{+}}\left(\frac{n-2}{2} u \frac{\partial u}{\partial \eta}-\frac{1}{2}(x \cdot \eta)|\nabla u|^{2}+\frac{\partial u}{\partial \eta}(x \cdot \nabla u)+\frac{n-2}{2 n}(x \cdot \eta) k u^{2 n /(n-2)}\right) d s_{x},
$$

where $B_{r}^{+}$is the upper half-ball of radius $r, \partial^{\prime \prime} B_{r}^{+}=\partial B_{r}^{+} \cap \mathbb{R}_{+}^{n}$, and $D(u)=\lim _{r \rightarrow 0} P(r, u)$. Under assumptions (1.7) on the growth of $u$ and (1.9) on the growth of $k$, we have the following characterization of the singular solutions of (1.1).

Theorem 1.1. If $u$ is a positive solution of (1.8), then $D(u) \leq 0$. Moreover, $D(u)=0$ if and only if zero is a removable singularity of $u$.

As a consequence of the characterization obtained in the last theorem, we are able to establish a result about the nonexistence of singular solutions for an elliptic problem with Dirichlet and Newmann conditions on the boundary (Theorem 1.2) and a result which is related to the asymptotic symmetry in a neighborhood of the origin (Theorem 1.3).

Now, under the same assumptions on the growth of $u$ and $k$ as in Theorem 1.1, we prove the following two results.

Theorem 1.2. Assume that $k(t x)$ is nonincreasing in $t$ for any unit vector $x \in \mathbb{R}^{n}$, and in $a$ neighborhood of the origin either $k(x) \equiv 1$ or (1.9) holds for $l \geq(n-2) / 2$. Let $\Omega$ be a smooth bounded domain in $\mathbb{R}^{n}$, such that $\Lambda \equiv \Omega \cap \mathbb{R}_{+}^{n}$ and $\partial^{\prime} \Lambda=\partial \Lambda \cap \partial \mathbb{R}_{+}^{n}$ are nonempty, and $x \cdot \eta>0$ for all $x \in \partial \Omega \cap \mathbb{R}_{+}^{n}$. Then, there are no positive smooth solutions of

$$
\begin{gathered}
\Delta u+k(x) u^{(n+2) /(n-2)}=0 \quad \text { in } \Lambda, \\
u=0 \quad \text { in } \partial^{\prime \prime} \Lambda, \\
\frac{\partial u}{\partial \eta}=0 \quad \text { in } \partial^{\prime} \Lambda \backslash\{0\},
\end{gathered}
$$

where $\partial^{\prime \prime} \Lambda=\partial \Lambda \cap \mathbb{R}_{+}^{n}$.

Theorem 1.3. If $u$ is a positive solution of problem (1.8), then

$$
u(x)=\bar{u}(|x|)(1+o(1))
$$

as $x \rightarrow 0$, where $\bar{u}(r)=f_{\partial^{\prime \prime} B_{r}^{+}} u(x) d s_{x}$ denotes the integral average of $u$ on $\partial^{\prime \prime} B_{r}^{+}$. 
We want to mention that if $v$ is a positive smooth solution of

$$
\begin{gathered}
\Delta v+\bar{k}(x) v^{(n+2) /(n-2)}=0 \quad \text { in } \mathbb{R}_{+}^{n}, \\
\frac{\partial v}{\partial \eta}=0 \quad \text { in } \partial \mathbb{R}_{+}^{n},
\end{gathered}
$$

such that $v(x)=O\left(|x|^{2-n}\right)$ at infinity, then $v$ satisfies the following Kazdan-WarnerEscobar-type identity:

$$
\int_{\mathbb{R}_{+}^{n}}(x \cdot \nabla \bar{k}) v^{2 n /(n-2)} d x=0 .
$$

Conversely, Theorem 1.1 implies the following result of conformal geometry.

Theorem 1.4. Suppose that $\bar{k}(x)$ satisfies $\bar{k}(\infty)=\lim _{|x| \rightarrow+\infty} \bar{k}(x)>0$, and

$$
c_{1}|x|^{-(l+1)} \leq|\nabla \bar{k}(x)| \leq c_{2}|x|^{-(l+1)}
$$

for large $|x|, l \geq(n-2) / 2$, and $c_{1}, c_{2}$ positive constants. Let $v$ be a positive solution of problem (1.13) satisfying the inequality

$$
v(x) \leq c|x|^{(2-n) / 2},
$$

for large $|x|$ and some positive constant c. If the identity (1.14) holds then the conformal metric $\bar{g}=v^{4 /(n-2)} d x^{2}$ can be realized as a smooth metric in $\mathbf{S}_{+}^{n}$. Furthermore, if $v$ is such that the conformal metric cannot be realized as a smooth metric on $\mathbf{S}_{+}^{n}$, then there exist positive constants $c_{1}$ and $c_{2}$ such that

$$
c_{1}|x|^{(2-n) / 2} \leq v(x) \leq c_{2}|x|^{(2-n) / 2}
$$

for large $|x|, x_{n}>0$.

Observe that Theorem 1.1 says that if $k(x)$ is a positive continuous function which satisfies (1.9) for some $l \geq(n-2) / 2$, then, for any positive solution $u$ of (1.8) which satisfies (1.7), we have $D(u)=0$ if and only if 0 is a removable singularity of $u$. We note that the hypothesis $l \geq(n-2) / 2$ in Theorem 1.1 is optimal.

In fact, assume that $k(x)=k(|x|)$ is radially symmetric, bounded between two positive constants and satisfies

$$
k(r)=1-A r^{l}+R(r)
$$

in a neighborhood of zero for some $A>0, l>0, R(r)=o\left(r^{l}\right)$, and $R^{\prime}(r)=o\left(r^{l-1}\right)$ when $r \rightarrow 0$. Let $u(r, \alpha)$ be the unique solution of the initial value problem

$$
\begin{gathered}
u^{\prime \prime}(r)+\frac{n-1}{r} u^{\prime}(r)+k(r) u^{(n+2) /(n-2)}=0 \quad \text { in }[0,1], \\
u(0)=\alpha, \quad u^{\prime}(0)=0 .
\end{gathered}
$$

Then, as a consequence of [4, Theorem 1.6], we obtain the following theorem. 
Theorem 1.5. Assume that (1.18) holds for $l<(n-2) / 2$ and $k(r)$ is nonincreasing in $r$ for $0 \leq r \leq 1$. Then, there exists a sequence $\alpha_{j} \rightarrow+\infty$ such that $u\left(r, \alpha_{j}\right)$ converges in $\mathbf{C}_{\mathrm{loc}}^{2}\left(B_{1}^{+}\right)$to a positive and singular solution $u$ of

$$
\begin{gathered}
\Delta u+k(x) u^{(n+2) /(n-2)}=0 \quad \text { in } B_{1}^{+}, \\
\frac{\partial u}{\partial \eta}=0 \quad \text { in } \partial^{\prime} B_{1}^{+} \backslash\{0\},
\end{gathered}
$$

such that $D(u)=0$.

This paper is organized as follows. In Section 2, we will study some properties of the smooth solutions of problem (1.8) and, when $k$ is a constant, we discuss the behavior of solutions $u$ of problem (1.8) or (1.13) with a nonremovable singularity at the origin. In Section 3, we will prove the theorem of characterization of removable singularities of solutions of problem (1.8). In Section 4, we will prove the theorem of asymptotic symmetry of solutions of problem (1.8) in a neighborhood of the origin and the other applications of Theorem 1.1.

\section{Preliminaries}

First we recall without proof a Pohozaev-type identity derived in [6], and two theorems which follow from the results of Caffarelli et al. in [2].

Theorem 2.1 (Pohozaev identity). Let $\Omega \subset \mathbb{R}^{n}(n \geq 3)$ be a bounded domain with smooth boundary; $u: \Omega \rightarrow \mathbb{R} \in \mathbf{C}^{2}(\Omega) ; f: \Omega \times \mathbb{R} \rightarrow \mathbb{R}$ continuous in $x, u ; F: \Omega \times \mathbb{R} \rightarrow \mathbb{R}$ defined by $F(x, u)=\int_{0}^{u} f(x, s) d s$. Suppose that $u$ is a solution of the equation $-\Delta u=f(x, u), x \in$ $\Omega$. Then

$$
\begin{aligned}
\int_{\Omega}\left(n F-\frac{n-2}{2} u f+\sum_{i} x^{i} F_{x^{i}}\right) d x \\
\quad=\int_{\partial \Omega}\left(\frac{n-2}{2} u \frac{\partial u}{\partial \eta}+\sum_{i} x^{i} \eta^{i}\left(F-\frac{1}{2}|\nabla u|^{2}\right)+\frac{\partial u}{\partial \eta} \sum_{i} x^{i} u_{i}\right) d s_{x}
\end{aligned}
$$

where $\eta$ denotes the normal outward unit vector in the boundary.

If $f(x, u)=k(x) u^{(n+2) /(n-2)}, u>0$, then the identity transforms into

$$
\begin{aligned}
& \frac{n-2}{2 n} \int_{\Omega}(x \cdot \nabla k) u^{2 n /(n-2)} d x \\
& \quad=\int_{\partial \Omega}\left(\frac{n-2}{2} u \frac{\partial u}{\partial \eta}-\frac{1}{2}(x \cdot \eta)|\nabla u|^{2}+\frac{\partial u}{\partial \eta}(x \cdot \nabla u)+\frac{n-2}{2 n}(x \cdot \eta) k u^{2 n /(n-2)}\right) d s_{x} .
\end{aligned}
$$

THeOREM 2.2. Let $u$ be a positive solution of (1.1) with a nonremovable isolated singularity. If $k \equiv 1$, then there exists a unique asymptotic constant $-(2 / n)((n-2) / n)^{n} \leq D_{\infty}<0$ and a radial singular solution $h(r)=h_{D}(\log r) / r^{(n-2) / 2}$ such that

$$
u(x)=h(|x|)(1+o(1)) \quad \text { as } x \longrightarrow 0 .
$$


684 Singular solutions of curvature-type equations

Theorem 2.3. Let $\lambda$ be a constant and let $u$ be a positive $\mathbf{C}^{2}$ solution of

$$
\Delta u+\lambda u^{(n+2) /(n-2)}=0 \quad \text { in } \mathbb{R}^{n} \backslash\{0\} .
$$

If the origin is an isolated nonremovable singularity, then $u$ is radially symmetric with respect to the origin.

Now, we will prove the results of this section. First observe the following proposition. Proposition 2.4. If $u(x)$ is a smooth solution of (1.8) in a neighborhood of the origin in $\overline{\mathbb{R}_{+}^{n}}$, then

$$
\int_{\partial^{\prime} B_{r}^{+}}\left(\frac{n-2}{2} u \frac{\partial u}{\partial \eta}-\frac{1}{2}(x \cdot \eta)|\nabla u|^{2}+\frac{\partial u}{\partial \eta}(x \cdot \nabla u)+\frac{n-2}{2 n}(x \cdot \eta) k u^{2 n /(n-2)}\right) d s_{x}=0,
$$

where $\partial^{\prime} B_{r}^{+}=\partial B_{r}^{+} \cap \partial \mathbb{R}_{+}^{n}$.

A straightforward calculation shows the following proposition.

Proposition 2.5. If $k(x) \equiv 1$, the function

$$
u_{0}(x)=\left(\frac{n-2}{2}\right)^{(n-2) / 2}|x|^{(2-n) / 2}
$$

is a solution of problem (1.8).

Set $\Omega=B_{r}^{+}$. The Pohozaev identity implies that

$$
\begin{aligned}
\frac{n-2}{2 n} \int_{B_{r}^{+}}(x \cdot \nabla k) u^{2 n /(n-2)} d x=P(r, u)+\int_{\partial^{\prime} B_{r}^{+}} & \frac{n-2}{2} u \frac{\partial u}{\partial \eta}-\frac{1}{2}(x \cdot \eta)|\nabla u|^{2} \\
& \left.+\frac{\partial u}{\partial \eta}(x \cdot \nabla u)+\frac{n-2}{2 n}(x \cdot \eta) k u^{2 n /(n-2)}\right) d s_{x}
\end{aligned}
$$

where $P(r, u)$ was defined in (1.10).

Proposition 2.6. If $u(x)$ is a solution of $(1.8)$ with $k(x) \equiv 1$, then $P(r, u)$ is a constant independent of $r$.

Proof. Observe that $\partial u / \partial \eta=0$ in $\partial^{\prime} B_{r}^{+} \backslash\{0\},(x \cdot \eta)=0$ on $\partial^{\prime} B_{r}^{+}=\partial B_{r}^{+} \cap \partial \mathbb{R}_{+}^{n}$, and $(x \cdot \eta)=r$ on $\partial^{\prime \prime} B_{r}^{+}=\partial B_{r}^{+} \cap \mathbb{R}_{+}^{n}$. Then for $0<s<r$, we have

$$
\frac{n-2}{2 n} \int_{B_{r}^{+} \backslash B_{s}^{+}}(x \cdot \nabla k) u^{2 n /(n-2)} d x=P(r, u)-P(s, u),
$$

and therefore $P(r, u)=P(s, u)$.

Proposition 2.7. Let $u(x)$ be a smooth solution of (1.8) in a neighborhood of the origin in $\overline{\mathbb{R}_{+}^{n}}$, then

(i) if $k(x) \equiv 1, P(r, u)=0$;

(ii) if $k(x)$ is smooth and nonconstant, then $D(u)=\lim _{r \rightarrow 0} P(r, u)=0$. 
Proof. Item (i) is a consequence of the Pohozaev identity and Proposition 2.4. From (1.10), we have

$$
P(r, u)=\int_{\partial^{\prime \prime} B_{r}^{+}}\left(\frac{n-2}{2} u \frac{\partial u}{\partial \eta}-\frac{1}{2} r|\nabla u|^{2}+\frac{\partial u}{\partial \eta}(x \cdot \nabla u)+\frac{n-2}{2 n} r k u^{2 n /(n-2)}\right) d s_{x} .
$$

By the assumption on $u$ and $k$, we conclude that $D(u)=\lim _{r \rightarrow 0} P(r, u)=0$.

Proposition 2.8. Let $k(x) \equiv 1$. If $u(x)$ is a positive solution of (1.8) and zero is a nonremovable singular point of $u$, then

$$
u(x)=u_{0}(x)(1+o(1))
$$

as $x \rightarrow 0$; furthermore $P(r, u)<0$.

Proof. Since $\partial u / \partial \eta=0$ in $\partial^{\prime} B_{r}^{+} \backslash\{0\}$, we can extend $u$ to a function $\hat{u}$ defined in the ball $B_{r}$ by

$$
\hat{u}(y, t)= \begin{cases}u(y, t) & \text { if } t \geq 0, \\ u(y,-t) & \text { if } t<0,\end{cases}
$$

where $y \in \mathbb{R}^{n-1},|(y, t)|<r$. It follows from Theorem 2.2 that

$$
\hat{u}(x)=u_{0}(x)(1+o(1))
$$

as $x \rightarrow 0$. Consequently

$$
u(x)=u_{0}(x)(1+o(1))
$$

as $x \rightarrow 0$.

Using this equality and Proposition 2.5, we get

$$
\begin{aligned}
P(r, u) & =\int_{\partial^{\prime \prime} B_{r}^{+}}\left(\frac{n-2}{2} u \frac{\partial u}{\partial \eta}-\frac{1}{2} r|\nabla u|^{2}+\frac{\partial u}{\partial \eta}(x \cdot \nabla u)+\frac{n-2}{2 n} r u^{2 n /(n-2)}\right) d s_{x} \\
& \leq c \int_{\partial^{\prime \prime} B_{r}^{+}}\left(-\frac{1}{2}\left(\frac{n-2}{2}\right)^{n}|x|^{1-n}+\frac{n-2}{2 n}\left(\frac{n-2}{2}\right)^{n}|x|^{1-n}\right) d s_{x} \\
& =c \int_{\partial^{\prime \prime} B_{r}^{+}}\left(\left(\frac{n-2}{2}\right)^{n}|x|^{1-n}\left(-\frac{1}{n}\right)\right) d s_{x}<0,
\end{aligned}
$$

where $c$ is a positive constant.

Proposition 2.9. Let $\lambda$ be a constant and let $u$ be a positive $\mathbf{C}^{2}$ solution of

$$
\begin{gathered}
\Delta u+\lambda u^{(n+2) /(n-2)}=0 \quad \text { in } \mathbb{R}_{+}^{n} \\
\frac{\partial u}{\partial \eta}=0 \quad \text { in } \partial \mathbb{R}_{+}^{n} \backslash\{0\} .
\end{gathered}
$$

If the origin is a nonremovable isolated singularity, then $u$ is radially symmetrical with respect to the origin. 
Proof. Using the extension of $u$, as in the previous proposition, the result is a consequence of Theorem 2.3.

\section{Removable singularities}

In this section, we prove that the quantity $D(u)$ characterizes the positive singular solutions of problem (1.8), which helps us to study the asymptotic behavior of such solutions and prove a result of nonexistence. An important application of this characterization to conformal geometry says that if $u$ is a positive solution to problem (1.13) for which the identity (1.14) holds, then the conformal metric $u^{4 /(n-2)}|d x|^{2}$ is smooth in $\mathbf{S}_{+}^{n}$.

From now on, assume that $k \in \mathbf{C}^{1}\left(B_{r}^{+}\right)$is bounded between two positive constants and satisfies inequalities (1.9). Furthermore, we will suppose that if $u$ is a positive solution of (1.8), then there exists a positive constant $c$ such that $u$ satisfies inequality (1.7) in a neighborhood of the origin.

Theorem 1.1 is a consequence of the following five lemmas which will be proved later in this section. We start by establishing a Harnack inequality on the upper half-sphere of radius $r$.

Lemma 3.1. Let u be a positive solution of problem (1.8). Then there exists a positive constant $C$ such that there exists the estimates

$$
\max _{|x|=r} u \leq C \min _{|x|=r} u, \quad|\nabla u(x)| \leq C|x|^{-1} u(x)
$$

for $|x| \leq 1 / 2$ with $x_{n} \geq 0$.

Using inequalities (3.1), we prove the following estimates.

LEMMA 3.2. Let $u$ be a positive solution of (1.8) and let $w(t)=\bar{u}(r) r^{(n-2) / 2}$, where $t=\log r$, $r \in(0,1]$ and, $\bar{u}(r)=f_{\partial^{\prime \prime} B_{r}^{+}} u$ is the integral average of $u$ on $\partial^{\prime \prime} B_{r}^{+}$. Then $w$ satisfies

$$
\left(\frac{n-2}{2}\right)^{2} w-c_{1} w^{(n+2) /(n-2)} \leq w_{t t} \leq\left(\frac{n-2}{2}\right)^{2} w-c_{2} w^{(n+2) /(n-2)}
$$

for $t \leq 0$, where $c_{1}$ and $c_{2}$ are two positive constants.

The following two lemmas are technical; they will be used in the proof of Lemma 3.5, which is one of the fundamental steps in the proof of Theorem 1.1.

Lemma 3.3. Let $u$ be a positive solution of (1.8). Assume that $D(u) \geq 0$. Then

$$
\varliminf_{x \rightarrow 0} u(x)|x|^{(n-2) / 2}=0 .
$$

Lemma 3.4. Let $u$ and $w$ be as in Lemma 3.2. Let $r_{i}=e^{t_{i}}$, where $t_{i}$ is a sequence of negative numbers such that $t_{i} \rightarrow-\infty$, and $w\left(t_{i}\right) \rightarrow 0$ as $i \rightarrow+\infty$, with $w^{\prime}\left(t_{i}\right)=0$. Then $v_{i}(y)=$ $r_{i}^{(n-2) / 2} u\left(r_{i} y\right)$ is such that $\left(v_{i}(e)\right)^{-1} v_{i}(y)$, with $e=(1,0, \ldots, 0)$, converges uniformly to the harmonic function $h(y)=(1 / 2)|y|^{2-n}+1 / 2$. 
Lemma 3.5. Suppose that $k(x)$ satisfies (1.9) for some $l \geq(n-2) / 2$. Let $u$ and $w$ be as in Lemma 3.2. If $D(u) \geq 0$, then $\lim _{t \rightarrow-\infty} w(t)=0$ and there exists $T$ such that $w^{\prime}(t)>0$ for $t \leq T$.

Proof of Theorem 1.1. Let $u$ be a positive solution of (1.8). Assuming that $D(u) \geq 0$, Lemma 3.5 implies that $w(t) \rightarrow 0$ as $t \rightarrow-\infty$, and there exists $T$ such that $w^{\prime}(t)>0$ for $t \leq T$. For any $\epsilon>0,|\epsilon|<1, T$ can be chosen such that

$$
w_{t t}-\left(\frac{n-2}{2}-\epsilon\right)^{2} w \geq 0 \quad \text { for } t \leq T .
$$

Integrating this differential inequality, we have for $t \leq T$,

$$
w(t) \leq w(T) \exp \left[\left(\frac{n-2}{2}-\epsilon\right)(t-T)\right] .
$$

Since $\bar{u}(r)=w(t) r^{(2-n) / 2}$, where $r=e^{t}$, applying the Harnack inequality (3.1), we have that for any $\epsilon>0,|\epsilon|<1$, there exists $r_{0}=e^{T}$ such that

$$
\begin{aligned}
u(x) \leq C \bar{u}|x| & =C w(t)|x|^{(2-n) / 2} \\
& \leq C w(T) \exp \left[\left(\frac{n-2}{2}-\epsilon\right)(t-T)\right]|x|^{(2-n) / 2} \\
& =C w(T) r^{-\epsilon} \exp \left[-\left(\frac{n-2}{2}-\epsilon\right) T\right] \\
& \leq c(\epsilon) r^{-\epsilon}
\end{aligned}
$$

for $r=|x|<r_{0}$. Hence $u \in L^{p}\left(B_{r_{0}}\right)$ for $p$ large. By estimates of linear elliptic equations (see [7]), the function $u(x)$ is smooth at the origin. Therefore, zero is a removable singularity and so, Theorem 1.1 follows.

Now, we will prove the lemmas used in the proof of Theorem 1.1.

Proof of Lemma 3.1. Let $v_{r}(x)=u(r x)$ for each $r \in(0,3 / 4]$ and for $1 / 2 \leq|x| \leq 1$. A straightforward calculation shows that $v_{r}$ satisfies

$$
\begin{gathered}
\Delta v_{r}(x)+b_{r}(x) v_{r}(x)=0 \quad \text { in } \frac{1}{2} \leq|x| \leq 1, x_{n} \geq 0, \\
\frac{\partial v_{r}}{\partial \eta}=0, \quad \frac{1}{2} \leq|x| \leq 1, x_{n}=0
\end{gathered}
$$

where $b_{r}(x)=r^{2} k(r x)(u(r x))^{4 /(n-2)}$. Due to inequality $(1.7), b_{r}(x)$ is bounded for $1 / 2 \leq$ $|x| \leq 1$.

Set

$$
\hat{v}_{r}= \begin{cases}u(r(y, t)) & \text { if } t \geq 0 \\ u(r(y,-t)) & \text { if } t<0\end{cases}
$$

where $y \in \mathbb{R}^{n-1},|(y, t)|<1$.

After applying the Harnack inequality and the estimate of the gradient for $\hat{v}_{r}$, (see [4]), the conclusion of Lemma 3.1 follows. 
Proof of Lemma 3.2.

$$
w(t)=\frac{2 r^{(n-2) / 2}}{n \alpha_{n} r^{n-1}} \int_{\partial^{\prime \prime} B_{r}^{+}} u(x) d s_{x}=\frac{2 r^{-n / 2}}{n \alpha_{n}} \int_{\partial^{\prime \prime} B_{r}^{+}} u(x) d s_{x}
$$

where $\alpha_{n}$ is the volume of the unit ball $B_{1}$ in $\mathbb{R}^{n}$. Hence

$$
\begin{aligned}
w_{t}= & \left(\frac{2 r}{n \alpha_{n}}\right)\left(-\frac{n}{2} r^{-(n+2) / 2} \int_{\partial^{\prime \prime} B_{r}^{+}} u(x) d s_{x}+r^{-n / 2} \frac{d}{d r} \int_{\partial^{\prime \prime} B_{1}^{+}} u(r y) r^{n-1} d s_{y}\right) \\
= & -\frac{n r^{(n-2) / 2}}{2} \int_{\partial^{\prime \prime} B_{r}^{+}} u(x) d s_{x}+\frac{2 r^{(2-n) / 2}}{n \alpha_{n}}\left[\int_{\partial^{\prime \prime} B_{1}^{+}}\left(\nabla u(r y) \cdot y r^{n-1}\right) d s_{y}\right] \\
& +\frac{2(n-1) r^{(n-2) / 2}}{n \alpha_{n}}\left[\int_{\partial^{\prime \prime} B_{1}^{+}} u(r y) d s_{y}\right] \\
= & \frac{n-2}{2} w+\frac{2 r^{(2-n) / 2}}{n \alpha_{n}}\left(\int_{B_{r}^{+}} \Delta u(x) d x-\int_{\partial^{\prime} B_{r}^{+}} \nabla u(x) \cdot \eta d s_{x}\right) \\
= & \frac{n-2}{2} w+\frac{2 r^{(2-n) / 2}}{n \alpha_{n}} \int_{B_{r}^{+}} \Delta u(x) d x .
\end{aligned}
$$

Taking again the derivative with respect to $t$, we get

$$
\begin{aligned}
w_{t t}= & \frac{n-2}{2} w_{t}+\frac{(2-n) r^{(2-n) / 2}}{2 n \alpha_{n}} \int_{B_{r}^{+}} \Delta u(x) d x \\
& +\frac{2 r^{(2-n) / 2}}{n \alpha_{n}}\left(\frac{d}{d r} \int_{B_{r}^{+}} \Delta u(x) d x\right) \frac{d r}{d t} \\
= & \left(\frac{n-2}{2}\right)^{2} w-r^{(n+2) / 2} f_{\partial^{\prime \prime} B_{r}^{+}} k(x) u^{(n+2) /(n-2)} d s_{x} .
\end{aligned}
$$

By the assumption $k(x)$ is bounded between two positive constants; furthermore (3.1) implies the existence of two positive constants $c_{3}$ and $c_{4}$ such that

$$
c_{3}(\bar{u}(r))^{(n+2) /(n-2)} \leq u(x)_{|x|=r}^{(n+2) /(n-2)} \leq c_{4}(\bar{u}(r))^{(n+2) /(n-2)} .
$$

Applying these estimates in (3.11), the conclusion of Lemma 3.2 follows.

Proof of Lemma 3.3. Suppose that there exists a positive constant $c_{0}$ such that

$$
c_{0} \leq u(x)|x|^{(n-2) / 2} \leq c
$$

for $0<|x| \leq 1 / 2, x_{n}>0$. Let $r_{j}$ be a sequence of positive numbers such that $r_{j} \rightarrow 0$ as $j \rightarrow+\infty$. Set $v_{j}(x)=u\left(r_{j} x\right) r_{j}^{(n-2) / 2}$. By (3.13), we have

$$
c_{0} \leq v_{j}(x)|x|^{(n-2) / 2} \leq c,
$$


and $v_{j}$ satisfies

$$
\begin{gathered}
\Delta v_{j}+k\left(r_{j} x\right) v_{j}^{(n+2) /(n-2)}=0 \quad \text { in } \mathbb{R}_{+}^{n}, \\
\frac{\partial v_{j}}{\partial \eta}=0 \quad \text { in } \partial \mathbb{R}_{+}^{n} \backslash\{0\} .
\end{gathered}
$$

Since $v_{j}$ is uniformly bounded in any compact subset of $\mathbb{R}_{+}^{n}$, by elliptic estimates there exists a subsequence of $v_{j}$ (still denoted by $v_{j}$ ) such that $v_{j}$ converges in $\mathbf{C}_{\mathrm{loc}}^{2}\left(\mathbb{R}_{+}^{n}\right)$ to a solution $v$ of

$$
\begin{gathered}
\Delta v+k(0) v^{(n+2) /(n-2)}=0 \quad \text { in } \mathbb{R}_{+}^{n}, \\
\frac{\partial v}{\partial \eta}=0 \quad \text { in } \partial \mathbb{R}_{+}^{n} \backslash\{0\}, \\
v(x) \geq c_{1}|x|^{-(n-2) / 2} .
\end{gathered}
$$

Proposition 2.8 implies that $P(1, v)<0$; furthermore, by $(1.10)$, we get

$$
\begin{aligned}
P\left(r_{j}, u\right)= & \int_{\partial^{\prime \prime} B_{r_{j}}^{+}}\left(\frac{n-2}{2} u(x) \frac{\partial u}{\partial \eta}-\frac{1}{2} r_{j}|\nabla u|^{2}+r_{j}\left|\frac{\partial u}{\partial \eta}\right|^{2}+\frac{n-2}{2 n} r_{j} k(x) u^{2 n /(n-2)}\right) d s_{x} \\
= & \int_{\partial^{\prime \prime} B_{1}^{+}}\left(\frac{n-2}{2} u\left(r_{j} y\right) \frac{\partial u}{\partial \eta} r_{j}^{n-1}-\frac{1}{2} r_{j}^{n}|\nabla u|^{2}\right. \\
& \left.+r_{j}^{n}\left|\frac{\partial u}{\partial \eta}\right|^{2}+\frac{n-2}{2 n} r_{j}^{n} k\left(r_{j} y\right) u^{2 n /(n-2)}\right) d s_{y} \\
= & P\left(1, v_{j}\right) .
\end{aligned}
$$

Then

$$
0 \leq D(u)=\lim _{r_{j} \rightarrow 0} P\left(r_{j}, u\right)=\lim _{j \rightarrow+\infty} P\left(1, v_{j}\right)=P(1, v)<0
$$

yields a contradiction. This finishes the proof of Lemma 3.3.

Proof of Lemma 3.4. Let $x=r_{i} y$. The Harnack inequality on any compact set of $\mathbb{R}_{+}^{n}$ implies that

$$
\begin{aligned}
v_{i}(y) & =r_{i}^{(n-2) / 2} u(x) \leq r_{i}^{(n-2) / 2} \max u(x) \leq C r_{i}^{(n-2) / 2} \min u(x) \\
& \leq C r_{i}^{(n-2) / 2} \min _{|x|=r_{i}} u(x) \leq C r_{i}^{(n-2) / 2} \bar{u}\left(r_{i}\right)=C w\left(t_{i}\right) .
\end{aligned}
$$

Therefore, $v_{i}(y)$ uniformly converges to 0 in any compact subset of $\mathbb{R}_{+}^{n}$. Therefore, $z_{i}(y)=$ $\left(v_{i}(e)\right)^{-1} v_{i}(y)$, with $e=(1,0, \ldots, 0)$, uniformly converges to a harmonic function $h(y)$ with a possible singularity at 0 . By Liouville's theorem (see [1]), $h(y)=a|y|^{2-n}+b$. Since $h(e)=1$, we have $a+b=1$. 
Set $\bar{g}_{i}(r)=r^{(n-2) / 2} \bar{z}_{i}(y)=c\left(r r_{i}\right)^{(n-2) / 2} \bar{u}\left(r_{i} r\right)$, where $c=\left(v_{i}(e)\right)^{-1}$ and $r=|y|$. Then $\bar{g}_{i}(r)=c w(t)$ and $\bar{g}_{i}{ }^{\prime}(r)=c w^{\prime}(t)(1 / r)$, with $t=\log \left(r r_{i}\right)$. Then $\bar{g}_{i}{ }^{\prime}(1)=c w^{\prime}\left(t_{i}\right)=0$. Hence, $(d / d r)\left(h(r) r^{(n-2) / 2}\right)=0$ in $r=1$, where $g(r)=h(r) r^{(n-2) / 2}=\lim _{i \rightarrow+\infty} \bar{g}_{i}(r)$. Calculating $g^{\prime}(1)$, we obtain that $a=b$, which completes the proof of Lemma 3.4.

Proof of Lemma 3.5. We will prove by contradiction that $\lim _{t \rightarrow-\infty} w(t)=0$. Otherwise, by Lemma 3.3, there exists a sequence $\left\{t_{i}\right\}$ of local minima of $w$ with $\lim _{i \rightarrow+\infty} t_{i}=-\infty$ and $\lim _{i \rightarrow+\infty} w\left(t_{i}\right)=0$. The first inequality of (3.2) implies that there exists $\epsilon_{0}>0$ such that $w_{t t}>0$ if $w \leq \epsilon_{0}$. Then there exists $t_{i}^{*}<t_{i}<\bar{t}_{i}$ such that $w\left(t_{i}^{*}\right)=w\left(\bar{t}_{i}\right)=\epsilon_{0} ; w_{t}<0$ in $\left[t_{i}^{*}, t_{i}\right)$ and $w_{t}>0$ in $\left(t_{i}, \bar{t}_{i}\right]$. Since $\bar{t}_{i}<t_{i-1}$, then $\lim _{i \rightarrow+\infty} \bar{t}_{i}=-\infty$. Under these conditions, we will prove the following inequalities:

$$
\frac{2}{n-2} \log \frac{w(t)}{w\left(t_{i}\right)}-c_{1} \leq t_{i}-t \leq \frac{2}{n-2} \log \frac{w(t)}{w\left(t_{i}\right)}+c_{2}
$$

for $t \in\left[t_{i}^{*}, t_{i}\right]$, and

$$
\frac{2}{n-2} \log \frac{w(t)}{w\left(t_{i}\right)}-c_{1} \leq t-t_{i} \leq \frac{2}{n-2} \log \frac{w(t)}{w\left(t_{i}\right)}+c_{2}
$$

for $t \in\left[t_{i}, \bar{t}_{i}\right]$.

First, consider the function

$$
h(t)=w_{t}^{2}-\left(\frac{n-2}{2}\right)^{2} w^{2}+c w^{2 n /(n-2)}
$$

for $t \in\left[t_{i}^{*}, t_{i}\right]$, where $c=((n-2) / n) c_{1}$ and $c_{1}$ is the same constant of inequalities (3.2). Then

$$
h^{\prime}(t)=2 w_{t}\left[w_{t t}-\left(\frac{n-2}{2}\right)^{2} w+c_{1} w^{(n+2) /(n-2)}\right] \leq 0
$$

and $h(t) \geq h\left(t_{i}\right)$, that is,

$$
w_{t}^{2}-g(w) \geq-g\left(w\left(t_{i}\right)\right)
$$

where $g(w)=\left[((n-2) / 2)^{2} w^{2}-c w^{2 n /(n-2)}\right]$ and we use $w_{t}\left(t_{i}\right)=0$. Integrating the previous inequality, we find that

$$
t_{i}-t \leq \int_{w\left(t_{i}\right)}^{w(t)} \frac{d w}{\sqrt{g(w)-g\left(w\left(t_{i}\right)\right)}}
$$

By scaling $w(t) \equiv w=\eta w\left(t_{i}\right), \eta \in\left[1, w(t) / w\left(t_{i}\right)\right]$, we get

$$
\int_{w\left(t_{i}\right)}^{w(t)} \frac{d w}{\sqrt{g(w)-g\left(w\left(t_{i}\right)\right)}}=\int_{1}^{w(t) / w\left(t_{i}\right)} \frac{d \eta}{\sqrt{\bar{g}(\eta)-\bar{g}(1)}},
$$


where $\bar{g}(\eta)=((n-2) / 2)^{2} \eta^{2}-c w\left(t_{i}\right)^{4 /(n-2)} \eta^{2 n /(n-2)}$. Hence, for $\epsilon_{0}$ sufficiently small, we have

$$
\begin{aligned}
\bar{g}(\eta)-\bar{g}(1) & =\left(\eta^{2}-1\right)\left[\left(\frac{n-2}{2}\right)^{2}-c w\left(t_{i}\right)^{4 /(n-2)}\left(\frac{\eta^{2 n /(n-2)}-1}{\eta^{2}-1}\right)\right] \\
& \geq\left(\eta^{2}-1\right)\left[\left(\frac{n-2}{2}\right)^{2}-c_{3} w^{4 /(n-2)}\right]>0
\end{aligned}
$$

for $w \leq \epsilon_{0}$. Therefore

$$
\begin{aligned}
\int_{1}^{w(t) / w\left(t_{i}\right)} \frac{d \eta}{\sqrt{\bar{g}(\eta)-\bar{g}(1)}} & \leq \int_{1}^{w(t) / w\left(t_{i}\right)} \frac{d \eta}{\left\{\left(\eta^{2}-1\right)\left[((n-2) / 2)^{2}-c_{3} w^{4 / n-2}\right]\right\}^{1 / 2}} \\
& =\int_{1}^{w(t) / w\left(t_{i}\right)} \frac{d \eta}{((n-2) / 2)\left\{\left(\eta^{2}-1\right)\left[1-c_{4} w^{4 / n-2}\right]\right\}^{1 / 2}} \\
& \leq \frac{2}{n-2} \int_{1}^{w(t) / w\left(t_{i}\right)} \frac{d \eta}{\sqrt{\eta^{2}-1}}+c_{5} w\left(t_{i}\right)^{4 /(n-2)} \int_{1}^{w(t) / w\left(t_{i}\right)} \frac{\eta^{4 /(n-2)}}{\sqrt{\eta^{2}-1}} d \eta,
\end{aligned}
$$

where we used

$$
\alpha=c_{4} w^{4 /(n-2)}<1 \quad\left(\epsilon_{0} \text { sufficiently small }\right), \quad \sqrt{\frac{1}{1-\alpha}} \leq 1+\alpha\left(\lim _{n \rightarrow \infty} \sum_{q=1}^{n} \alpha^{q}\right) .
$$

By the last integral in the previous inequality, we get $\eta^{4 / n-2} \leq\left(w(t) / w\left(t_{i}\right)\right)^{4 /(n-2)-1} \eta$ in $\left[1, w(t) / w\left(t_{i}\right)\right]$. Hence

$$
\begin{aligned}
w\left(t_{i}\right)^{4 /(n-2)} \int_{1}^{w(t) / w\left(t_{i}\right)} \frac{\eta^{4 /(n-2)}}{\sqrt{\eta^{2}-1}} d \eta & \leq w\left(t_{i}\right)^{4 /(n-2)}\left(\frac{w(t)}{w\left(t_{i}\right)}\right)^{4 /(n-2)-1} \int_{1}^{w(t) / w\left(t_{i}\right)} \frac{\eta}{\sqrt{\eta^{2}-1}} d \eta \\
& =w\left(t_{i}\right)^{4 /(n-2)}\left(\frac{w(t)}{w\left(t_{i}\right)}\right)^{4 /(n-2)-1} \sqrt{\left(\frac{w(t)}{w\left(t_{i}\right)}\right)^{2}-1} \leq \epsilon_{0},
\end{aligned}
$$

and therefore

$$
\int_{1}^{w(t) / w\left(t_{i}\right)} \frac{d \eta}{\sqrt{\bar{g}(\eta)-\bar{g}(1)}} \leq \frac{2}{n-2} \log \frac{w(t)}{w\left(t_{i}\right)}+c_{6}
$$

for some positive constant $c_{6}$; this proves the first part of (3.20). To prove the second part, we consider the function

$$
\psi(t)=w_{t}^{2}-\left(\frac{n-2}{2}\right)^{2} w^{2}(t)
$$


for $t \in\left[t_{i}^{*}, t_{i}\right]$. By inequalities (3.2), we have

$$
\psi^{\prime}(t)=2 w_{t}\left[w_{t t}-\left(\frac{n-2}{2}\right)^{2} w(t)\right] \geq 0
$$

and therefore

$$
w_{t}^{2}-\left(\frac{n-2}{2}\right)^{2} w^{2}(t) \leq-\left(\frac{n-2}{2}\right)^{2} w^{2}\left(t_{i}\right),
$$

where we used $w_{t}\left(t_{i}\right)=0$. Integrating the previous inequality, we get

$$
\frac{n-2}{2}\left(t_{i}-t\right) \geq \int_{w\left(t_{i}\right)}^{w(t)} \frac{d w}{\sqrt{w^{2}(t)-w^{2}\left(t_{i}\right)}} .
$$

By scaling $w=\eta w\left(t_{i}\right), \eta \in\left[1, w(t) / w\left(t_{i}\right)\right]$, we have

$$
\begin{aligned}
& \int_{w\left(t_{i}\right)}^{w(t)} \frac{d w}{\sqrt{w^{2}(t)-w^{2}\left(t_{i}\right)}} \\
& \quad=\int_{1}^{w(t) / w\left(t_{i}\right)} \frac{d \eta}{\sqrt{\eta^{2}-1}}=\log \left(\sqrt{\left(\frac{w(t)}{w\left(t_{i}\right)}\right)^{2}-1+\frac{w(t)}{w\left(t_{i}\right)}}\right) \geq \log \frac{w(t)}{w\left(t_{i}\right)}
\end{aligned}
$$

which completes the proof of inequalities (3.20). The proof of inequalities (3.21) is analogous and we skipped it. Evaluating these two expressions for $t_{i}^{*}$ and $\bar{t}_{i}$, respectively, we have

$$
\begin{aligned}
\frac{2}{n-2} \log \frac{w\left(t_{i}^{*}\right)}{w\left(t_{i}\right)}-c_{1} & \leq t_{i}-t_{i}^{*} \leq \frac{2}{n-2} \log \frac{w\left(t_{i}^{*}\right)}{w\left(t_{i}\right)}+c_{2} \\
\frac{2}{n-2} \log \frac{w\left(\bar{t}_{i}\right)}{w\left(t_{i}\right)}-c_{1} \leq \bar{t}_{i}-t_{i} & \leq \frac{2}{n-2} \log \frac{w\left(\bar{t}_{i}\right)}{w\left(t_{i}\right)}+c_{2}
\end{aligned}
$$

Hence,

$$
\frac{4}{n-2} \log \left(\frac{\epsilon_{0}}{w\left(t_{i}\right)}\right)-c \leq \bar{t}_{i}-t_{i}^{*}
$$

Let $r_{i}=e^{t_{i}}$ and $v_{i}(y)=r_{i}^{(n-2) / 2} u\left(r_{i} y\right)$. By Lemma 3.4, we have that if $|y|=1$ then $v_{i}(y)=$ $v_{i}(e)(1+o(1))$. Consequently,

$$
\begin{gathered}
u(x)=\bar{u}(r)(1+o(1)), \\
|\nabla u(x)|=-\bar{u}^{\prime}(r)(1+o(1))
\end{gathered}
$$


in $x=r_{i} y,|x|=r=r_{i}$. Using this result together with (3.17), we get

$$
\begin{aligned}
P(r, u)= & \int_{\partial^{\prime \prime} B_{1}^{+}}\left(\frac{n-2}{2} u(r y) \frac{\partial u}{\partial \eta} r^{n-1}-\frac{1}{2} r^{n}|\nabla u|^{2}+r^{n}\left|\frac{\partial u}{\partial \eta}\right|^{2}+\frac{n-2}{2 n} r^{n} k(r y) u^{2 n /(n-2)}\right) d s_{y} \\
= & \int_{\partial^{\prime \prime} B_{1}^{+}}\left(\frac{n-2}{2} \bar{u}(r) \bar{u}^{\prime}(r) r^{n-1}(1+o(1))^{2}+\frac{1}{2} r^{n}\left(\bar{u}^{\prime}(r)\right)^{2}(1+o(1))^{2}\right) d s_{y} \\
& +\int_{\partial^{\prime \prime} B_{1}^{+}}\left(\frac{n-2}{2 n} k(r y)\left(r^{(n-2) / 2} \bar{u}(r)(1+o(1))\right)^{2 n /(n-2)}\right) d s_{y} \\
= & \int_{\partial^{\prime \prime} B_{1}^{+}}\left[\left(\frac{1}{2} w^{\prime 2}\left(t_{i}\right)-\frac{1}{2}\left(\frac{n-2}{2}\right)^{2} w^{2}\left(t_{i}\right)\right)(1+o(1))\right] d s_{y} \\
& +\int_{\partial^{\prime \prime} B_{1}^{+}}\left[\left(\frac{n-2}{2 n} k\left(r_{i} y\right) w^{2 n /(n-2)}\left(t_{i}\right)\right)(1+o(1))\right] d s_{y} \\
= & \frac{\sigma_{n-1}}{2}\left[\frac{1}{2} w^{\prime 2}\left(t_{i}\right)-\frac{1}{2}\left(\frac{n-2}{2}\right)^{2} w^{2}\left(t_{i}\right)+\frac{n-2}{2 n} \bar{k}\left(t_{i}\right) w^{2 n /(n-2)}\left(t_{i}\right)\right](1+o(1))
\end{aligned}
$$

where $\sigma_{n-1} \equiv n \alpha_{n}$ is the volume of $\partial B_{1}$ in $\mathbb{R}^{n}$ and $\bar{k}(t)=f_{\partial^{\prime \prime} B_{r}^{+}} k$ with $r=e^{t}$. By the above equation, we obtain the following conclusions.

(1) Since $w^{\prime}\left(t_{i}\right)=0$ and $w\left(t_{i}\right) \rightarrow 0$ as $i \rightarrow+\infty$, then

$$
D(u)=\lim _{i \rightarrow+\infty} P\left(r_{i}, u\right)=0 .
$$

(2) $P\left(r_{i}, u\right) \leq c_{1}(n) w^{2}\left(t_{i}\right)<0$ for $\epsilon_{0}$ sufficiently small.

Hence,

$$
\begin{aligned}
w^{2}\left(t_{i}\right) & \leq c_{n}\left|P\left(r_{i}, u\right)\right| \\
& \leq c_{n}\left[\int_{B_{r_{i}^{+}}^{+} \backslash B_{r_{i}^{*}}^{+}}|x \cdot \nabla k(x)| u^{2 n / n-2} d x+\int_{B_{r_{i}^{*}}^{+}}|x \cdot \nabla k(x)| u^{2 n / n-2} d x\right] \equiv I_{1}+I_{2},
\end{aligned}
$$

where $r_{i}^{*}=e^{t_{i}^{*}}$. By the assumption, we have $|x \cdot \nabla k(x)| \leq c_{0}|x|^{(n-2) / 2}$. Since $u$ satisfies inequality (1.7), we obtain the following estimate:

$$
\left|I_{2}\right|=\left|c_{n} \int_{B_{r_{i}^{+}}^{+}}\right| x \cdot \nabla k(x)\left|u^{2 n /(n-2)} d x\right| \leq c r_{i}^{*(n-2) / 2}=c \exp \left(\frac{n-2}{2} t_{i}^{*}\right) .
$$

To calculate $I_{1}$, by the first inequality of (3.20),

$$
w(t) \leq c w\left(t_{i}\right) \exp \left[\frac{n-2}{2}\left(t_{i}-t\right)\right]
$$


Setting $\Lambda \equiv B_{r_{i}}^{+} \backslash B_{r_{i}^{*}}^{+}$, we have

$$
\begin{aligned}
\left|I_{1}\right| & =\left|c_{n} \int_{\Lambda}\right| x \cdot \nabla k(x)\left|u^{2 n /(n-2)} d x\right| \\
& \leq c_{0} \int_{\Lambda}|x|^{(n-2) / 2} u^{2 n /(n-2)} d x \\
& =c_{0} \int_{r_{i}^{*}}^{r_{i}}\left(\int_{\partial^{\prime \prime} B_{r}^{+}} r^{(n-2) / 2} u(x)^{2 n /(n-2)} d s_{x}\right) d r \\
& \leq \tilde{c}_{0} \int_{r_{i}}^{r_{i}^{*}} w(t)^{2 n /(n-2)} r^{(n-4) / 2} d r \\
& \leq \tilde{c} w^{2 n /(n-2)}\left(t_{i}\right) \exp \left(n t_{i}\right) \int_{t_{i}^{*}}^{t_{i}} \exp \left(-\frac{n+2}{2} t\right) d t \\
& \leq \hat{c} w^{2 n /(n-2)}\left(t_{i}\right) \exp \left(n t_{i}\right) \exp \left(-\frac{n+2}{2} t_{i}^{*}\right) .
\end{aligned}
$$

By the second inequality of (3.20), we get

$$
w\left(t_{i}\right) \leq \bar{c} w\left(t_{i}^{*}\right) \exp \left[\frac{n-2}{2}\left(t_{i}^{*}-t_{i}\right)\right] .
$$

Putting these two inequalities together, we have

$$
\left|I_{1}\right| \leq \zeta \epsilon_{0}^{2 n /(n-2)} \exp \left(\frac{n-2}{2} t_{i}^{*}\right)
$$

Then, by (3.42), we get

$$
w^{2}\left(t_{i}\right) \leq C \exp \left(\frac{n-2}{2} t_{i}^{*}\right)
$$

which implies $-\log w\left(t_{i}\right) \geq-C_{2}+((n-2) / 4)\left(-t_{i}^{*}\right)$; applying (3.38),

$$
\begin{aligned}
\bar{t}_{i}-t_{i}^{*} & \geq \frac{4}{n-2} \log \epsilon_{0}-\frac{4}{n-2} \log w\left(t_{i}\right)-c \\
& \geq \frac{4}{n-2}\left[-C_{2}+\frac{n-2}{4}\left(-t_{i}^{*}\right)\right]-\frac{4}{n-2} \log \left(\frac{1}{\epsilon_{0}}\right)-c \\
& =\frac{4}{n-2} \frac{n-2}{4}\left(-t_{i}^{*}\right)-c_{3} \log \left(\frac{1}{\epsilon_{0}}\right)
\end{aligned}
$$

that is,

$$
\bar{t}_{i} \geq-c_{3} \log \left(\frac{1}{\epsilon_{0}}\right)
$$

which yields a contradiction with the fact that $\lim _{i \rightarrow+\infty} \bar{t}_{i}=-\infty$. Lemma 3.5 has been proved. 


\section{Applications}

The theorems in this section are consequences of Theorem 1.1. Recall that Theorem 1.2 is a result of nonexistence of singular solutions, Theorem 1.3 is a result of asymptotic symmetry in a neighborhood of the origin, and Theorem 1.4 is a result of conformal geometry.

Proof of Theorem 1.2. Suppose that $u>0$ is a solution of (1.11). Let $s>0$; for (2.8) and the assumption $u=0$ in $\partial^{\prime \prime} \Lambda$, we have

$$
\frac{n-2}{2 n} \int_{\Lambda \backslash B_{s}^{+}}(x \cdot \nabla k) u^{2 n /(n-2)} d x=\int_{\partial^{\prime \prime} \Lambda} \frac{1}{2}(x \cdot \eta)|\nabla u|^{2} d s_{x}-P(s, u) ;
$$

taking the limit when $s \rightarrow 0$, we obtain

$$
\frac{1}{2} \int_{\partial^{\prime \prime} \Lambda}(x \cdot \eta)|\nabla u|^{2} d s_{x}=\frac{n-2}{2 n} \int_{\Lambda}(x \cdot \nabla k) u^{2 n /(n-2)} d x+D(u) \leq 0
$$

by the assumption for $k$ and Theorem 1.1. Since $x \cdot \eta>o$ in $\partial^{\prime \prime} \Lambda$, we conclude that $|\nabla u| \equiv$ 0 and $\partial u / \partial \eta=0$ in $\partial^{\prime \prime} \Lambda$. This implies $u \equiv 0$ in $\Lambda$, which yields a contradiction.

Proof of Theorem 1.3. Suppose that 0 is a nonremovable singularity of $u$, otherwise the result is obvious. By Theorem 1.1, $D(u)=\lim _{r \rightarrow 0} P(r, u)<0$. We claim that there exists a constant $c_{0}$ such that

$$
u(x) \geq c_{0}|x|^{(2-n) / 2}
$$

for $0<|x| \leq 1 / 2$. In fact, let $w(t)=\bar{u}(r) r^{(n-2) / 2}, t=\log r$. If the claim is false then $\underline{\lim }_{t \rightarrow-\infty} w(t)=0$. Therefore, $\overline{\lim }_{t \rightarrow-\infty} w(t)>0$, otherwise, $\lim _{t \rightarrow-\infty} w(t)=0$ and 0 must be a removable singularity of $u$. Then there exists a sequence of $t_{i} \rightarrow-\infty$ such that $w^{\prime}\left(t_{i}\right)=0$. Using the same argument as in Lemma 3.5, we have

$$
P\left(r_{i}, u\right)=\frac{\sigma_{n-1}}{2}\left[\frac{1}{2} w^{\prime 2}\left(t_{i}\right)-\frac{1}{2}\left(\frac{n-2}{2}\right)^{2} w^{2}\left(t_{i}\right)+\frac{n-2}{2 n} \bar{k}\left(t_{i}\right) w^{2 n /(n-2)}\left(t_{i}\right)\right](1+o(1)) .
$$

Then $D(u)=\lim _{i \rightarrow+\infty} P\left(r_{i}, u\right)=0$, which is a contradiction. Consequently (4.3) has been proved. Next, we prove that

$$
\begin{gathered}
u(x)=\bar{u}(|x|)(1+o(1)), \\
|\nabla u(x)|=-\frac{\partial \bar{u}}{\partial r}(|x|)(1+o(1))
\end{gathered}
$$

as $x \rightarrow 0$.

Suppose that there exists $\epsilon_{0}>0$ such that

$$
u\left(x_{i}\right) \geq \bar{u}\left(r_{i}\right)\left(1+\epsilon_{0}\right)
$$


for a sequence of points $x_{i}$, where $r_{i}=\left|x_{i}\right| \rightarrow 0$ as $i \rightarrow+\infty$. Let $v_{i}(x)=u\left(r_{i} x\right) r_{i}^{(n-2) / 2}$. Then, as in the proof of Lemma 3.3, there exists a subsequence (still denoted by $v_{i}$ ) that converges in $\mathbf{C}_{\text {loc }}^{2}\left(\mathbb{R}_{+}^{n}\right)$ to a solution $v$ of

$$
\begin{gathered}
\Delta v+k(0) v^{(n+2) /(n-2)}=0 \quad \text { in } \mathbb{R}_{+}^{n}, \\
\frac{\partial v}{\partial \eta}=0 \quad \text { in } \partial \mathbb{R}_{+}^{n} \backslash\{0\} .
\end{gathered}
$$

Now, by (4.3), $v(x) \geq c_{0}|x|^{(2-n) / 2}$, then $v(x)=v(|x|)$ (see Proposition 2.9). But,

$$
\bar{v}_{j}(1)=f_{\partial^{\prime \prime} B_{1}^{+}} v_{j}(x) d s_{x}=r_{j}^{(n-2) / 2} \bar{u}\left(r_{j}\right)
$$

Hence, by (4.7), if $|x|=1$,

$$
v_{j}(x)=u\left(r_{j} x\right) r_{j}^{(n-2) / 2} \geq \bar{u}\left(r_{j}\right) r_{j}^{(n-2) / 2}\left(1+\epsilon_{0}\right)=\bar{v}_{j}(1)\left(1+\epsilon_{0}\right) .
$$

Therefore, if $|x|=1$,

$$
v(x)=\lim _{j \rightarrow+\infty} v_{j}(x) \geq \lim _{j \rightarrow+\infty} \bar{v}_{j}(1)\left(1+\epsilon_{0}\right)=v(x)\left(1+\epsilon_{0}\right)
$$

yields a contradiction. This proves the asymptotic symmetry of $u$. Arguing similarly, suppose that there exists $\epsilon_{0}>0$ such that

$$
\left|\nabla u\left(x_{i}\right)\right| \geq-\bar{u}^{\prime}\left(r_{i}\right)\left(1+\epsilon_{0}\right)
$$

for a sequence of points $x_{i}$, where $r_{i}=\left|x_{i}\right| \rightarrow 0$ as $i \rightarrow+\infty$, we arrive at

$$
\begin{aligned}
|\nabla v(x)| & =\lim _{j \rightarrow+\infty}\left|\nabla v_{j}(x)\right| \\
& \geq-\lim _{j \rightarrow+\infty} r_{j}^{n / 2} \bar{u}^{\prime}\left(r_{j}\right)\left(1+\epsilon_{0}\right) \\
& =-\lim _{j \rightarrow+\infty} r_{j}^{(n-2) / 2} f_{\partial B_{r_{j}}^{+}}(\nabla u(x) \cdot x) d s_{x}\left(1+\epsilon_{0}\right) \\
& =\lim _{j \rightarrow+\infty} f_{\partial B_{1}^{+}}\left|\nabla v_{j}(y)\right| d s_{y}\left(1+\epsilon_{0}\right) \\
& =|\nabla v(x)|\left(1+\epsilon_{0}\right),
\end{aligned}
$$

where $|x|=1$. From this contradiction, (4.6) follows.

Proof of Theorem 1.4. Suppose that $v$ is a solution of (1.13) such that (1.14) holds. We want to prove that $v(x)=O\left(|x|^{2-n}\right)$ at infinity, which implies that the conformal metric $\bar{g}$ can be realized as a smooth metric in $\mathbf{S}_{+}^{n}$. Let

$$
u(y)=|y|^{2-n} v\left(\frac{y}{|y|^{2}}\right)
$$


be the Kelvin transformation of $v$. Since (1.13) is invariant under the Kelvin transformation, $u(y)$ satisfies

$$
\begin{gathered}
\Delta u(y)+k(y) u^{(n+2) /(n-2)}=0 \quad \text { in } \mathbb{R}_{+}^{n}, \\
\frac{\partial u}{\partial \eta}=0 \quad \text { in } \partial \mathbb{R}_{+}^{n} \backslash\{0\}, \\
u(y)=O\left(|y|^{2-n}\right) \quad \text { at infinity, }
\end{gathered}
$$

where $k(y)=\bar{k}\left(y /|y|^{2}\right)$.

Taking $x=y /|y|^{2}$, we have $|\nabla k(y)|=|\nabla \bar{k}(x)||y|^{-2}$. By (1.15), we see that

$$
c_{1}|y|^{l-1} \leq|\nabla k(y)| \leq c_{2}|y|^{l-1}
$$

in a neighborhood of zero. Due to inequality (1.16), $u(y)$ is bounded by $c|y|^{(2-n) / 2}$ in a neighborhood of the origin, where $c$ is a positive constant. Hence, $D(u)=\lim _{r \rightarrow 0} P(r, u)$ exists and $D(u) \leq 0$.

To apply the Pohozaev identity in $\mathbb{R}_{+}^{n} \backslash B_{r}^{+}$, observe that

$$
\int_{\mathbb{R}_{+}^{n} \backslash B_{r}^{+}}(y \cdot \nabla \bar{k}) u^{2 n /(n-2)} d y=\lim _{\gamma \rightarrow+\infty} \int_{B_{\gamma}^{+} \backslash B_{r}^{+}}(y \cdot \nabla \bar{k}) u^{2 n /(n-2)} d y=\lim _{\gamma \rightarrow+\infty} P(\gamma, u)-P(r, u) .
$$

Now, since $u(y)=O\left(|y|^{2-n}\right)$ at infinity, a straightforward calculation shows that $P(\gamma, u)$ $\rightarrow 0$ as $\gamma \rightarrow+\infty$. Therefore

$$
\int_{\mathbb{R}_{+}^{n} \backslash B_{r}^{+}}(y \cdot \nabla k) u^{2 n /(n-2)} d y=-P(r, u) .
$$

Making the change of variables $x=\left(y /|y|^{2}\right)$, with Jacobian determinant $-|x|^{-2 n}$, we observe that $\nabla k(y) \cdot y=-\nabla \bar{k}(x) \cdot x$; therefore

$$
\int_{\mathbb{R}_{+}^{n} \backslash B_{r}^{+}}(y \cdot \nabla k(y)) u^{2 n /(n-2)} d y=-\int_{|x| \leq 1 / r}(x \cdot \nabla \bar{k}(x)) v^{2 n /(n-2)}(x) d x,
$$

which implies, by (1.14) and (4.18), that

$$
D(u)=\int_{\mathbb{R}_{+}^{n}}(x \cdot \nabla \bar{k}(x)) v^{2 n /(n-2)}(x) d x=0 .
$$

Then, by Theorem 1.1, $u$ has a removable singularity at zero; since $v(x)=u\left(x /|x|^{2}\right)|x|^{2-n}$, we conclude that $v(x)=O\left(|x|^{2-n}\right)$ at infinity. Now suppose that $v$ is such that the conformal metric cannot be realized as a smooth metric in $S_{+}^{n}$. Let $u$ be the Kelvin transformation of $v$. Then $D(u)<0$ and $u$ has a nonremovable singularity at zero. Therefore there exists two positive constants $c_{1}$ and $c_{2}$ such that

$$
c_{1}|y|^{2-n} \leq u(y) \leq c_{2}|y|^{2-n}
$$


in a neighborhood of zero. Consequently,

$$
c_{1}|x|^{(2-n) / 2} \leq v(x) \leq c_{2}|x|^{(2-n) / 2}
$$

for large $|x|, x_{n}>0$. This finishes the proof of Theorem 1.4.

\section{Acknowledgments}

G. García and H. Yaker were supported by Colciencias with the Grant no. 1106-05-10283, CT-220-2000. G. García was supported by Universidad del Valle (Cali, Colombia).

\section{References}

[1] S. Axler, P. Bourdon, and W. Ramey, Harmonic Function Theory, Graduate Texts in Mathematics, vol. 137, Springer, New York, 1992.

[2] L. A. Caffarelli, B. Gidas, and J. Spruck, Asymptotic symmetry and local behavior of semilinear elliptic equations with critical Sobolev growth, Comm. Pure Appl. Math. 42 (1989), no. 3, 271-297.

[3] C.-C. Chen and C.-S. Lin, Estimates of the conformal scalar curvature equation via the method of moving planes, Comm. Pure Appl. Math. 50 (1997), no. 10, 971-1017.

[4] - On the asymptotic symmetry of singular solutions of the scalar curvature equations, Math. Ann. 313 (1999), no. 2, 229-245.

[5]_, Estimates of the conformal scalar curvature equation via the method of moving planes III, Comm. Pure Appl. Math. 53 (2000), no. 10, 611-646.

[6] Y. Y. Li, Prescribing scalar curvature on $S^{n}$ and related problems. I, J. Differential Equations 120 (1995), no. 2, 319-410.

[7] C. Morrey Jr. and B. Charles, Multiple Integrals in the Calculus of Variations, Die Grundlehren der mathematischen Wissenschaften, Band 130, Springer, New York, 1966.

Gonzalo García: Departamento de Matemáticas, Facultad de Ciencias, Universidad del Valle, Cali, Colombia

E-mail address: ggarcia@mafalda.univalle.edu.co

Hendel Yaker: Departamento de Matemáticas y Estadística, Facultad de Ingeniería, Universidad Icesi, Cali, Colombia

E-mail address: hyaker@icesi.edu.co 


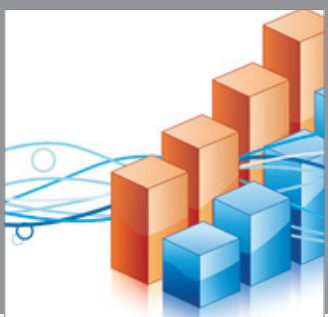

Advances in

Operations Research

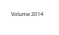

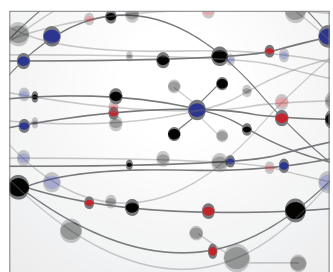

\section{The Scientific} World Journal
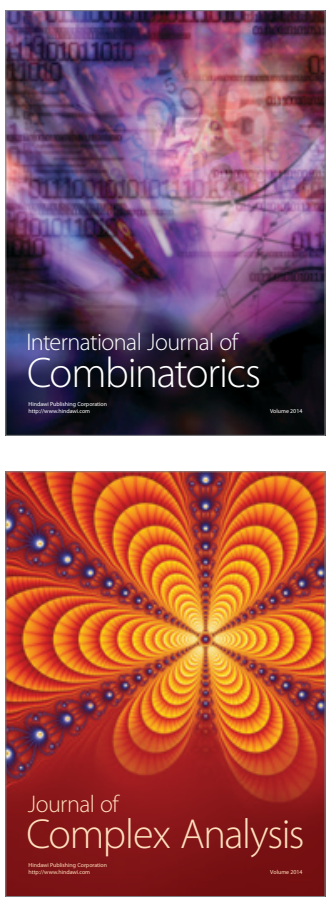

International Journal of

Mathematics and

Mathematical

Sciences
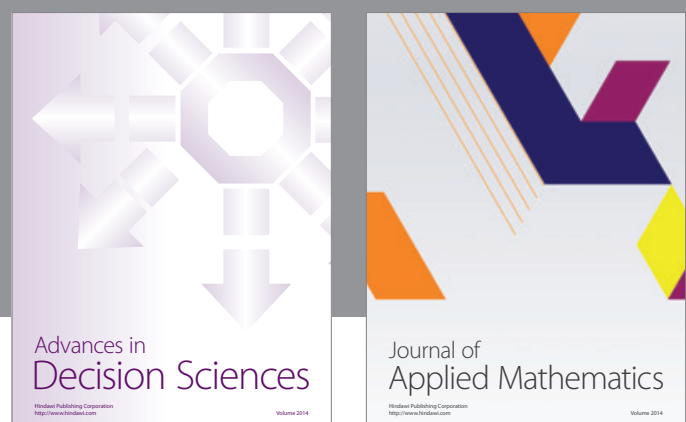

Journal of

Applied Mathematics
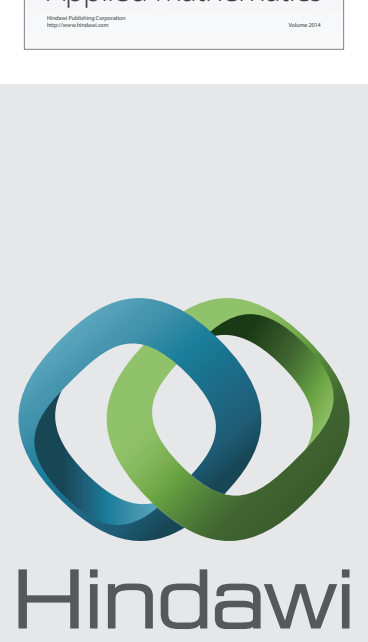

Submit your manuscripts at http://www.hindawi.com
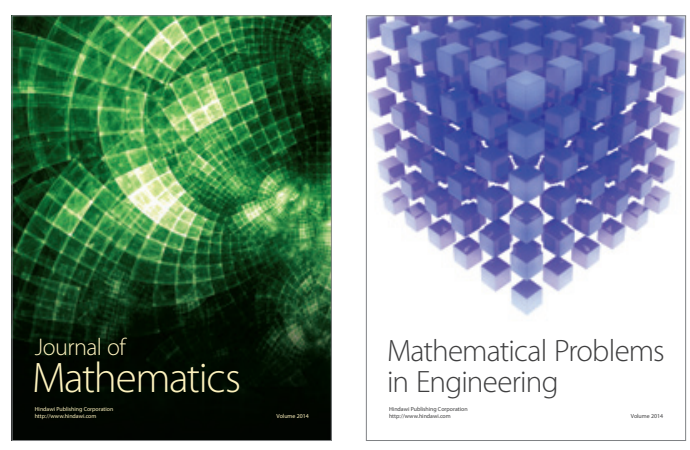

Mathematical Problems in Engineering
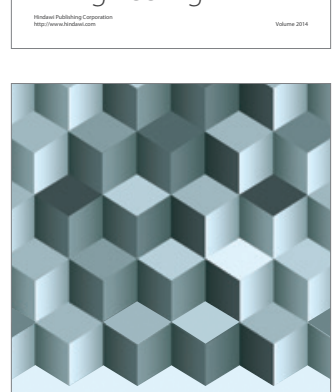

Journal of

Function Spaces
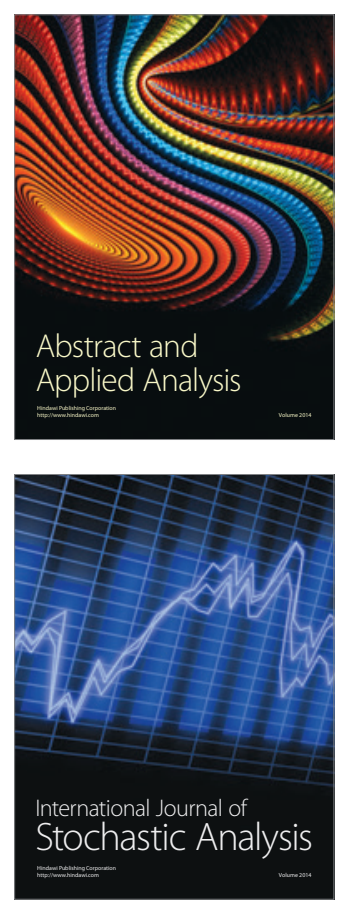

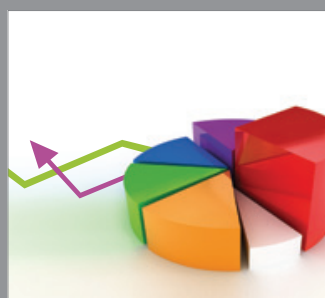

ournal of

Probability and Statistics

Promensencen
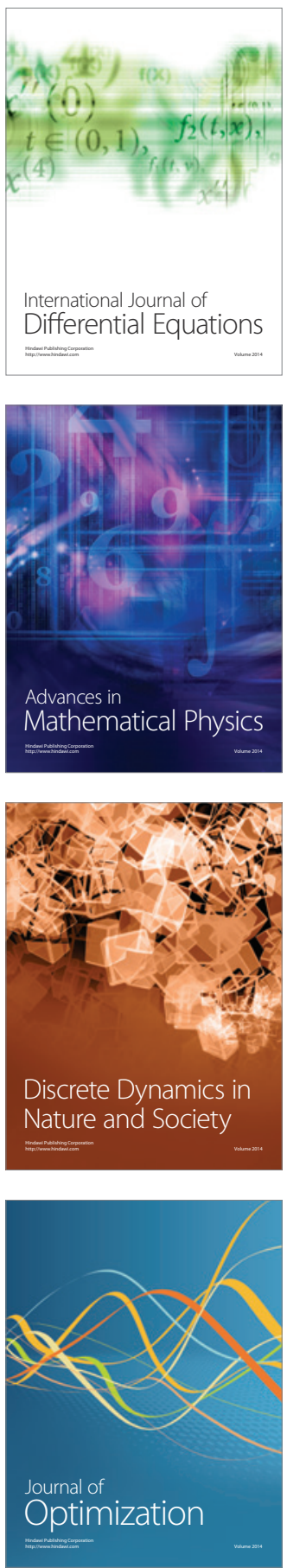